\title{
Simulation of Feedig Behavior of Sea Urchin Strongylocentrotus nudus
}

\author{
Yasuhiro HaYAKawa and Jiro KITTAKA*
}

(Accepted June 3, 1983)

\begin{abstract}
Three models for simulation of feeding behavior of sea urchins on algae were presented in comparison with results of tank experiments. It was assumed that the sea urchins fed on the algae most effectively in model-I, and less effectively in model-II. In both models their feeding behavior was characterized by a constant moving velocity of $10 \mathrm{~m} /$ day and a daily feeding rate of $7 \%$. On the other hand, velocity and direction of their movement were assumed to be stochastic variables in model-III. Feeding behavior was simulated in these models. A feeding pattern in model-III was similar to that of tank experiments. The density-dependent relationship was found between the sea urchins and the algae. Critical range for survival of the algal population was roughly estimated.
\end{abstract}

Many studies have been made on the relation between sea urchins and their food organisms, showing drastic effects of sea urchins grazing on benthic algal communities, ${ }^{1,2)}$ and even on sessile animals, ${ }^{3)}$ Nevertheless, one problem remains unsolved: How many sea urchins could have a grazing effect on the algae and to what extent? The sea urchin Strongylocentrotus nudus, which is common on the sublittoral rocks in the northern district of Japan, feeds on algae, especially the brown algae of genus Laminaria, Undaria and so on. It seems that the sea urchins do not have always an extirpating effect on these algae, which derives from the density-dependent relationship between the sea urchins and the algae. Furthermore, this relationship is essential in the cases of sea urchins transplantation from poor vegetative grounds to dense ones and of sea urchins culture.

The present paper describes some models for simulating the feeding behavior of the sea urchins to compare the resultant calculations with the experimental observations.

\section{Materials and Methods}

\section{Observation-I}

Sea urchins, starved previously for a week, were reared in small containers with the algae Undaria pinnatifida, Laminaria religiosa, and Ulva pertusa. Daily feeding rate was calculated by dividing wet weight of the algae eaten in one day by wet weight of the sea urchins. This rate was considered to be maximum, when hungry sea urchins were supplied with excess of food in a rather narrow area. Results are shown in Table 1 , indicating around a $7 \%$ feeding rate on the brown algae.

\section{Observation-II}

Feeding behavior concept included eating and moving of sea urchins. The former was characterized by a parameter of daily feeding rate just mentioned above, and the latter, by velocity and direction of sea urchins movements.

Two or four sea urchins starved for a week were kept in a cage of $5.12 \mathrm{~m}^{2}$ development surface $(1.6 \times 0.8 \mathrm{~m}, 0.8 \mathrm{~m}$ depth) with no food; their locations were observed every 30 minutes for 7 days in October, 1981 (Obs.-II-1), and for 3 days in May, 1982 (Obs.-II-2). Moving velocity and direction were known from changes in location. Examples of the velocity are shown in Fig. 1 together with water temperature and light intensity. Sometimes, a sea urchin with no food moved at a speed of about $100 \mathrm{~m} / \mathrm{day}$, but still was resting or staying for the greater part of the day. The daily geometric means of the velocity in Obs.-II-1 and Obs.-II-2 are given in the upper side of Fig. 2 together with the frequency in velocities and the total mean of all the moving sea urchin (velocity $>$ zero). Sums of velocity frequencies amounted to $49 \%$ in Obs.-II-1 and $34 \%$ in Obs.-II-2, since resting frequencies (velocity =zero) were $51 \%$ and $66 \%$, respectively. Total mean of sea urchins movements were $7.1 \mathrm{~m}$ /day and $13.4 \mathrm{~m} /$ day, but mean moving distances the

* School of Fisheries Sciences, Kitasato University, Sanriku, Kesen, Iwate 022-01, Japan（早川康博・埛高 二郎: 北里大学水産学部)。 
Table 1. Rearing experiment to evaluate daily feeding rate (July 11-November 12, 1980)

\begin{tabular}{ccccccccc}
\hline $\begin{array}{c}\text { Kind of } \\
\text { animal }\end{array}$ & $\begin{array}{c}\text { No. of } \\
\text { animals }\end{array}$ & $\begin{array}{c}\text { Average } \\
\text { body wt. } \\
(\mathrm{g})\end{array}$ & $\begin{array}{c}\text { No. of } \\
\text { rearing } \\
\text { days }\end{array}$ & $\begin{array}{c}\text { Kind of } \\
\text { food }\end{array}$ & $\begin{array}{c}\text { Daily } \\
\text { feeding } \\
\text { rate }(\%)\end{array}$ & $\begin{array}{c}\text { Rate of feeding } \\
\text { amount }(\%) \\
\text { daytime }\end{array}$ & $\begin{array}{c}\text { Water } \\
\text { night }\end{array}$ & $\begin{array}{c}\text { temp. } \\
\left({ }^{\circ} \mathrm{C}\right)\end{array}$ \\
\hline Sea urchin & 1 & 34.2 & 14 & Undaria & 7.27 & 43 & 57 & 18.5 \\
& 3 & 26.1 & 15 & Laminaria & 7.62 & 43 & 57 & 18.5 \\
& 2 & 19.3 & 20 & Ulva & 2.15 & 34 & 66 & 16.4 \\
& 1 & 29.5 & 20 & Ulva & 1.09 & 36 & 64 & 16.4 \\
\hline
\end{tabular}

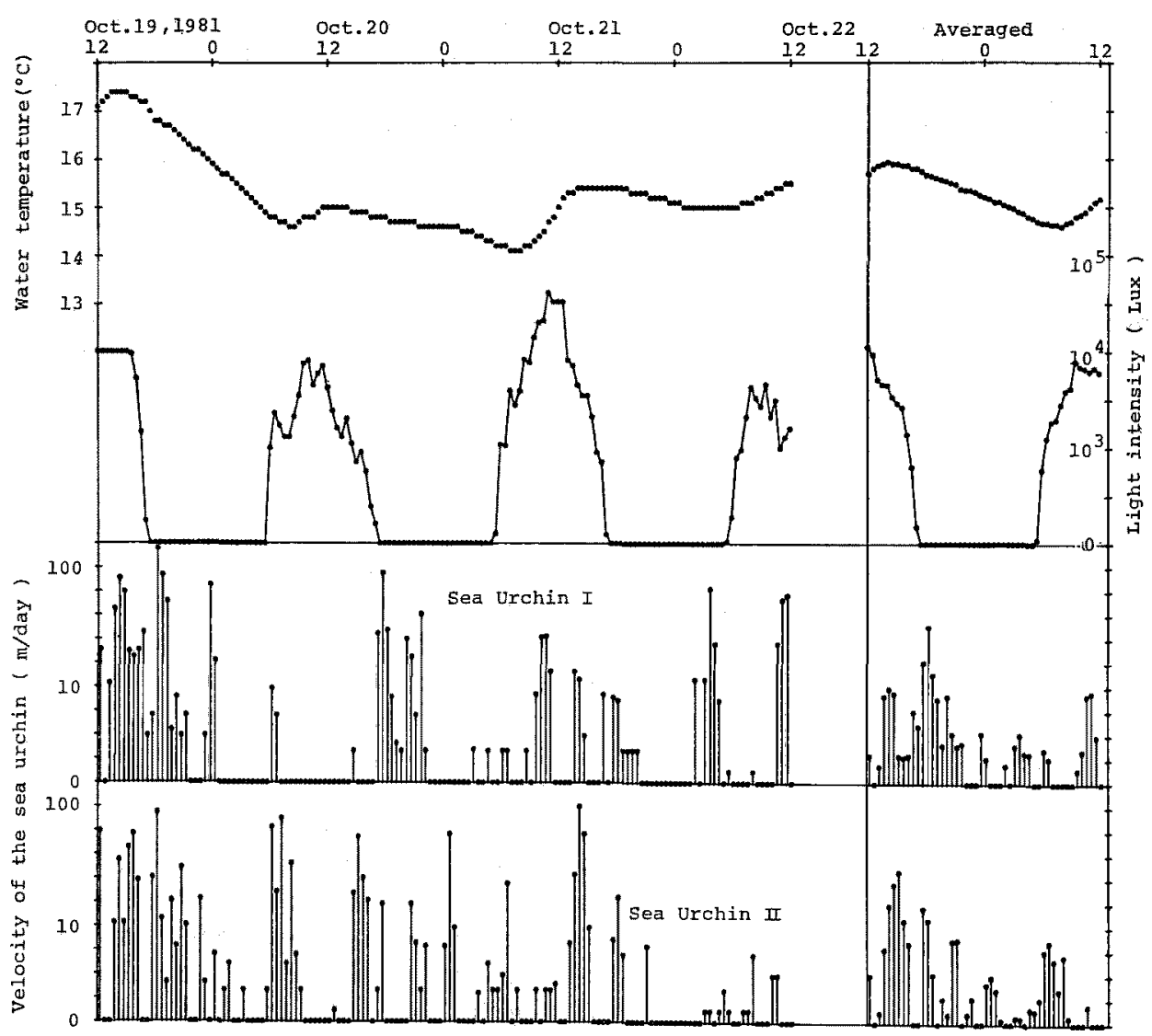

Fig. 1. Velocity of sea urchins supplied with no food.

Sea urchin I (test dia., $69.1 \mathrm{~mm}$, wet weight, $131 \mathrm{~g}$ ).

Sea urchin II $(47.4 \mathrm{~mm}, 49 \mathrm{~g})$.

Diurnal cycle of averaged velocity on the right hand. Note that velocity and light intensity are on the logarithmic scale except for zero.

sea urchins covered in a day amounted to $5 \mathrm{~m}$ and $9 \mathrm{~m}$ respectively, considering the resting periods and arithmetic mean of velocities. Relative directions, given by differences between the present direction and the previous one, were calculated too. Frequencies in relative directions are shown in the lower side of Fig. 2, suggesting random distribution.

\section{Observation-III}

Sea urchins were kept with the algae Undaria pinnatifida uniformly distributed in the cage. Each alga was about $10 \mathrm{~cm}$ long and its weight was about $0.8 \mathrm{~g}$. The eaten algae number was counted everyday for a few weeks. The observations were made with 2 sea urchins $(0.4$ individuals per $\mathrm{m}^{2}$ ) and 269 algae ( 52.5 individuals per $\mathrm{m}^{2}$ ), to which Obs.-III-1 refers. Four sea 

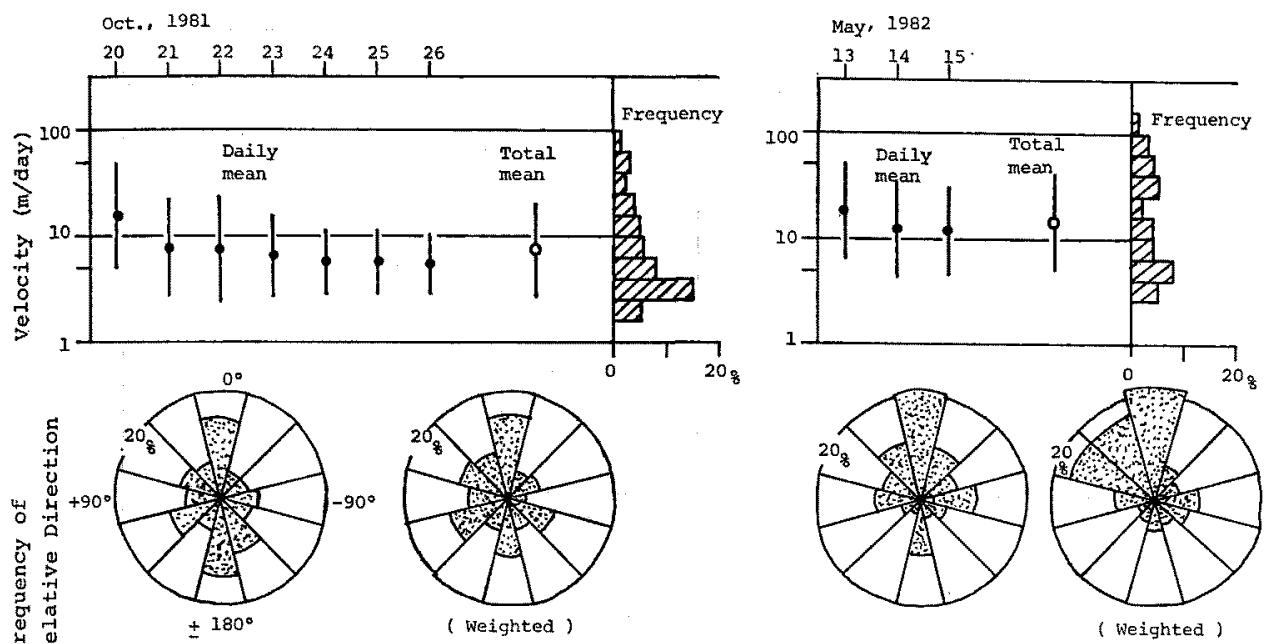

Fig. 2. Mean velocity and relative direction of moving sea urchins in Obs.-II-1 (Oct., 1981) and Obs.-II-2 (May, 1982).

Obs.-II-1; two sea urchins of $69.1 \mathrm{~mm}$ and $47.4 \mathrm{~mm}$ in test diameter.

Obs.-II-2; four of $73.0 \mathrm{~mm}, 53.8 \mathrm{~mm}, 42.8 \mathrm{~mm}$ and $42.5 \mathrm{~mm}$ in test diameter.

Daily geometric mean (solid circle) with standard deviation, total geometric mean (blank circle), and frequency of velocity are in the upper half. Frequency of relative dirction and resultants of velocity and dircetion are in the lower.

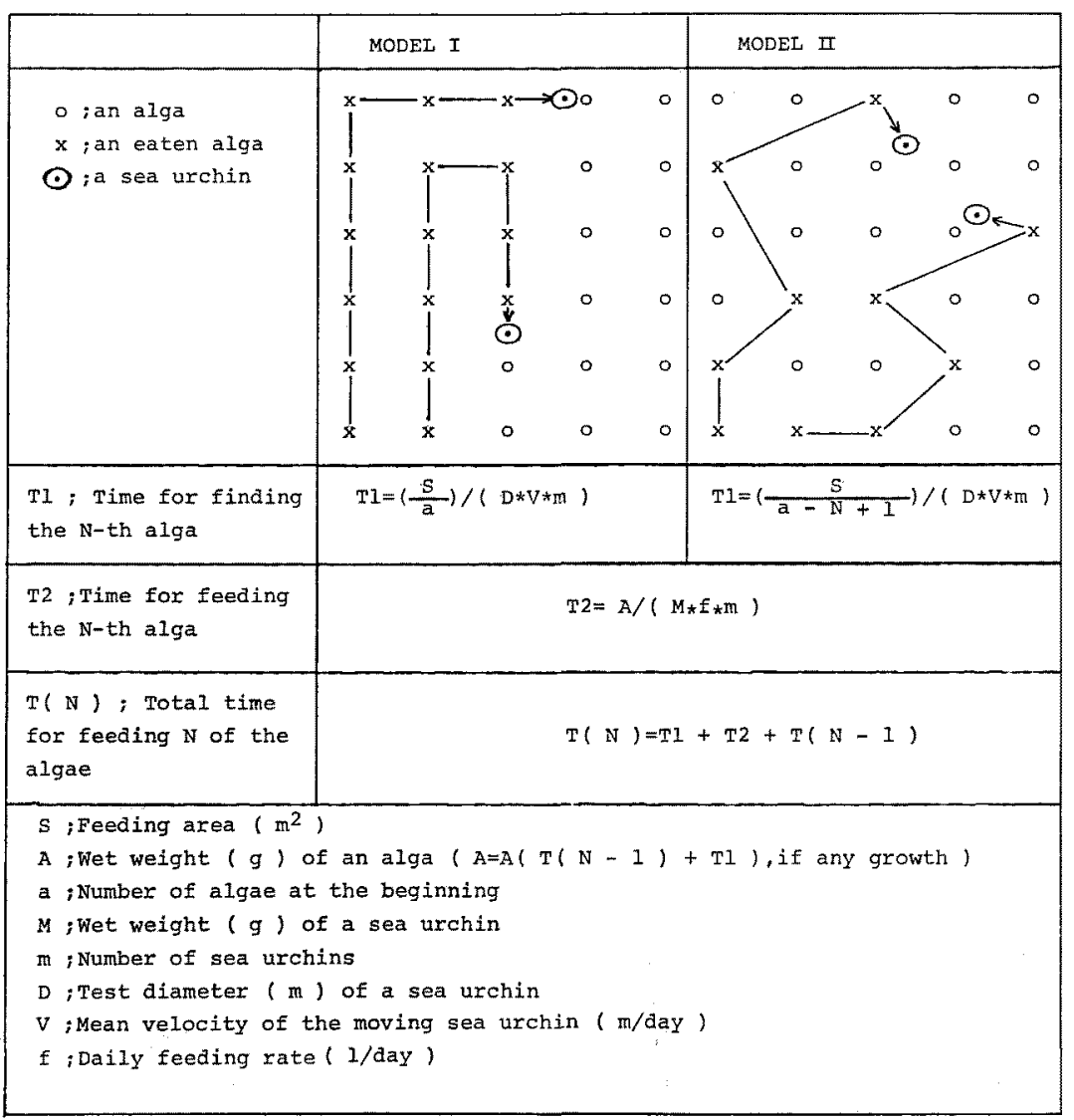

Fig. 3. Illustration of model-I and model-II. 


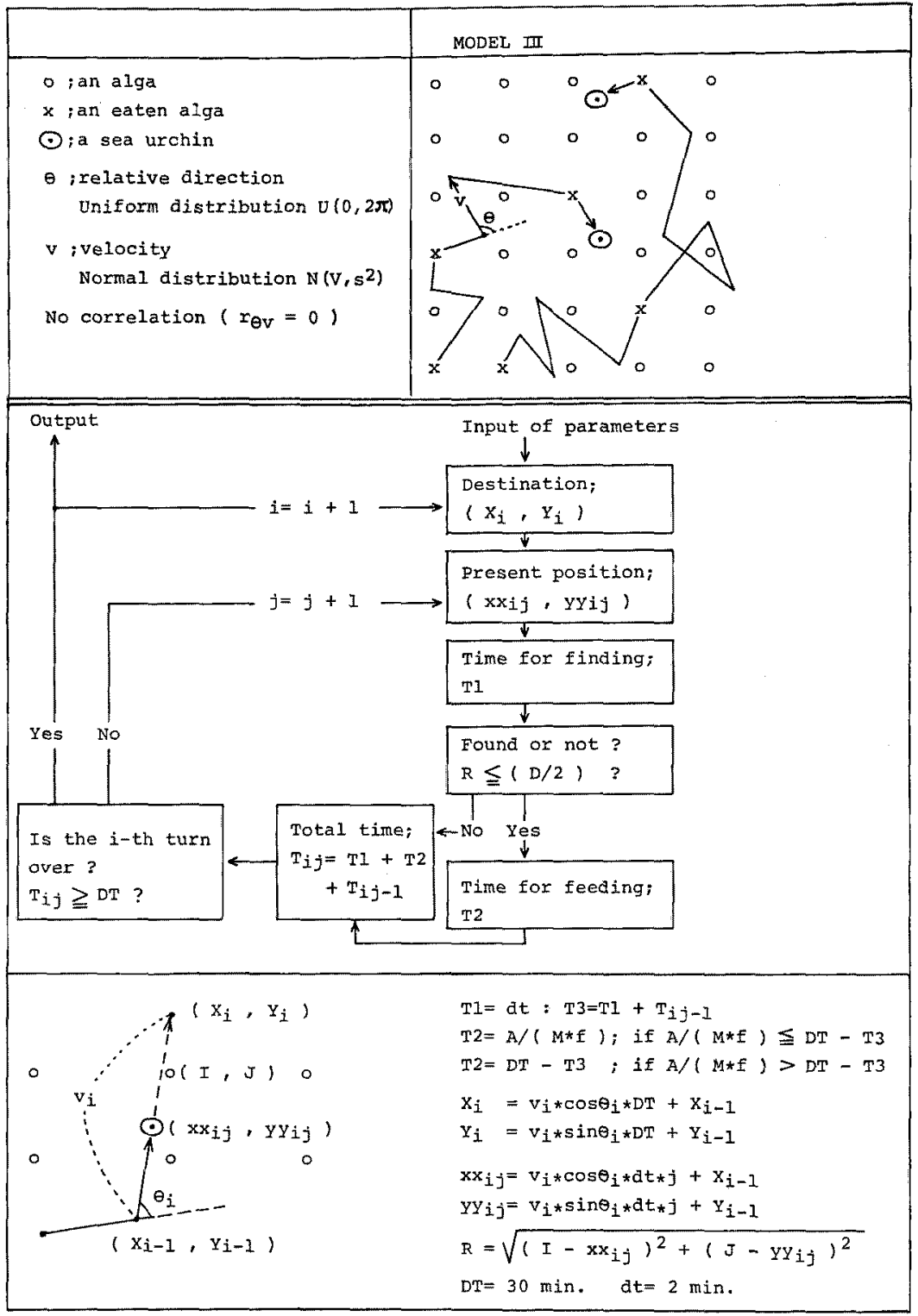

Fig. 4. Illustration and flow chart of model-III.

urchins with 134 algae were observed in Obs.-III-2. These results will be shown later, being compared with those of the simulation model.

\section{Models}

\section{Model-I and Model-II}

In model-I, the sea urchin moves so efficiently that it has only to cover the average area occupied by an alga in order to find any one of the algae. Thus, time for finding the $N$-th alga $(T 1)$ is given by

$$
T I=S /(a \cdot D \cdot V \cdot m)
$$

wherein $S$ is feeding area, $a$ is number of algae at the beginning, $D$ is test diameter of a sea urchin, $V$ is mean velocity of the sea urchins movements and $m$ is number of sea urchins. If these parameters are fixed, $T l$ has a constant value.

In model-II, $T I$ is inversely proportional to number of the remaining algae. Thus, $T 1$ is given by

$$
T I=S /((a-N+1) \cdot(D \cdot V \cdot m)) .
$$

Time for finding the first alga in model-II equals that in model-I, but the sea urchins in model-II must move and cover the whole area to find the last one. So, $T I$ in model-II is a function of $N$; 


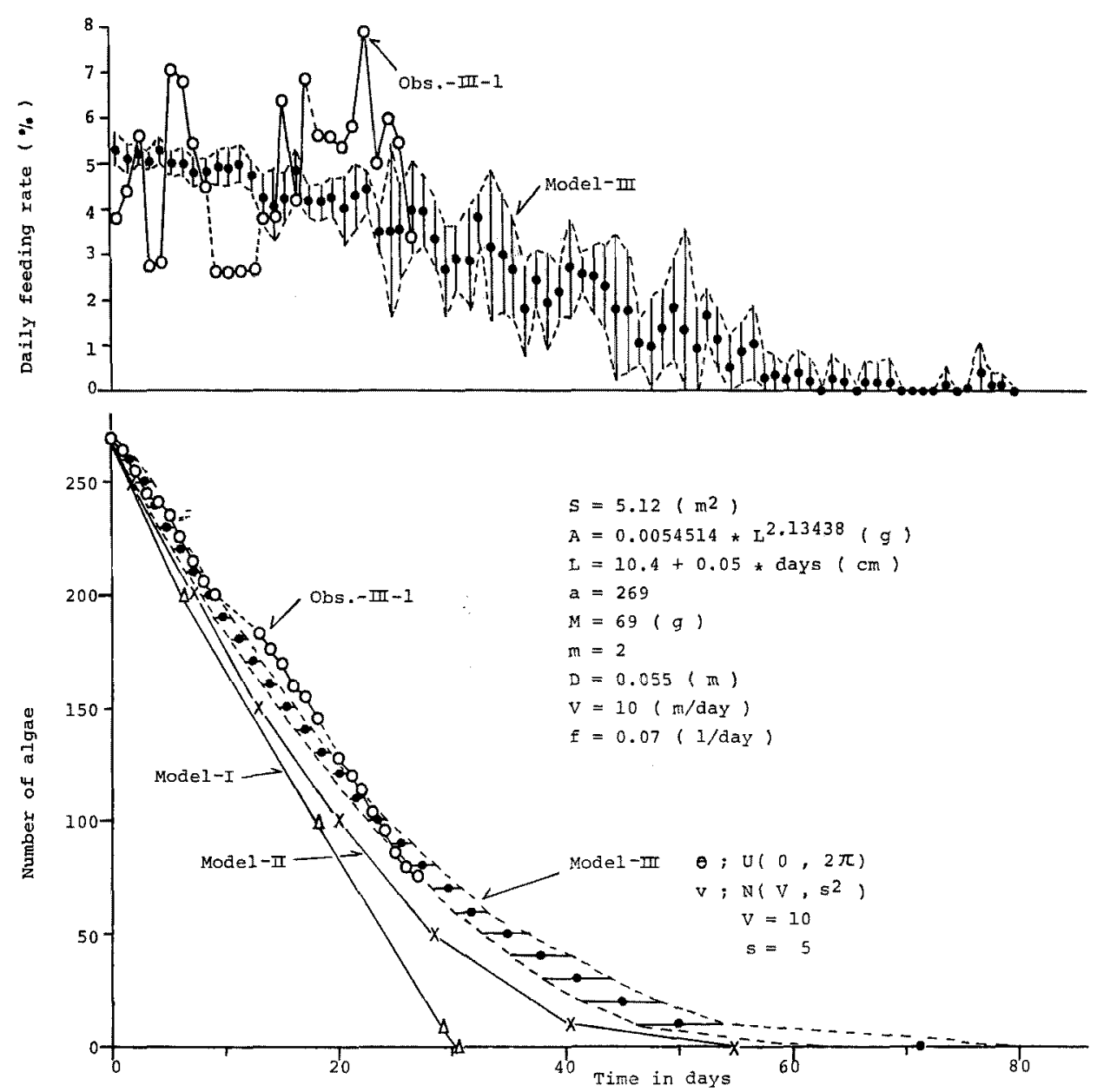

Fig. 5. Comparison of observation (Obs.-III-1; Dec., 1981) with model-I, model-II, and model-III. In model-III, averages of five calculations are plotted with their ranges.

number of the eaten algae.

In both models, $T 2$ providing time for eating the $N$-th alga is given by the following;

$$
T 2=A /(M \cdot f \cdot m)
$$

wherein $A$ is wet weight of an alga, $M$ is wet weight of a sea urchin, and $f$ is daily feeding rate. $T 2$ is constant if these parameters are fixed. Of course, $T 2$ depends on time if any growth of algae occurs.

Total time for finishing feeding (or finding and eating) on the $N$ algae is given by

$$
T(N)=T 1+T 2+T(N-1)
$$

wherein $T(N)$ is total time for finishing feeding on the $N$ algae, and a function of number of the eaten algae. Fig. 3 illustrates model-I and modelII.

\section{Model-III}

In model-III, the movement of the sea urchins is described by two stochastic variables; velocity $(v)$ and relative direction $(\theta)$. It is assumed that $v$ follows normal distribution $\mathbf{N}\left(V, s^{2}\right)$, wherein $V$ is mean velocity and $s$ is its standard deviation, and that $\theta$ is distributed uniformly; $\mathbf{U}(0,2 \pi)$. There is no correlation between $v$ and $\theta$. The sea urchin finds the algae when it encounters them within its test diameter. After finding the algae, it immediately starts eating them at a speed of its daily feeding rate. When it finished eating, it begins to move again. In the meanwhile, remaining algae grow slightly according to the given growth curve. Fig. 4 shows outlines of model-III and the flow chart. This simulation was performed on the screen of a personal computer (SHARP MZ-80B). 


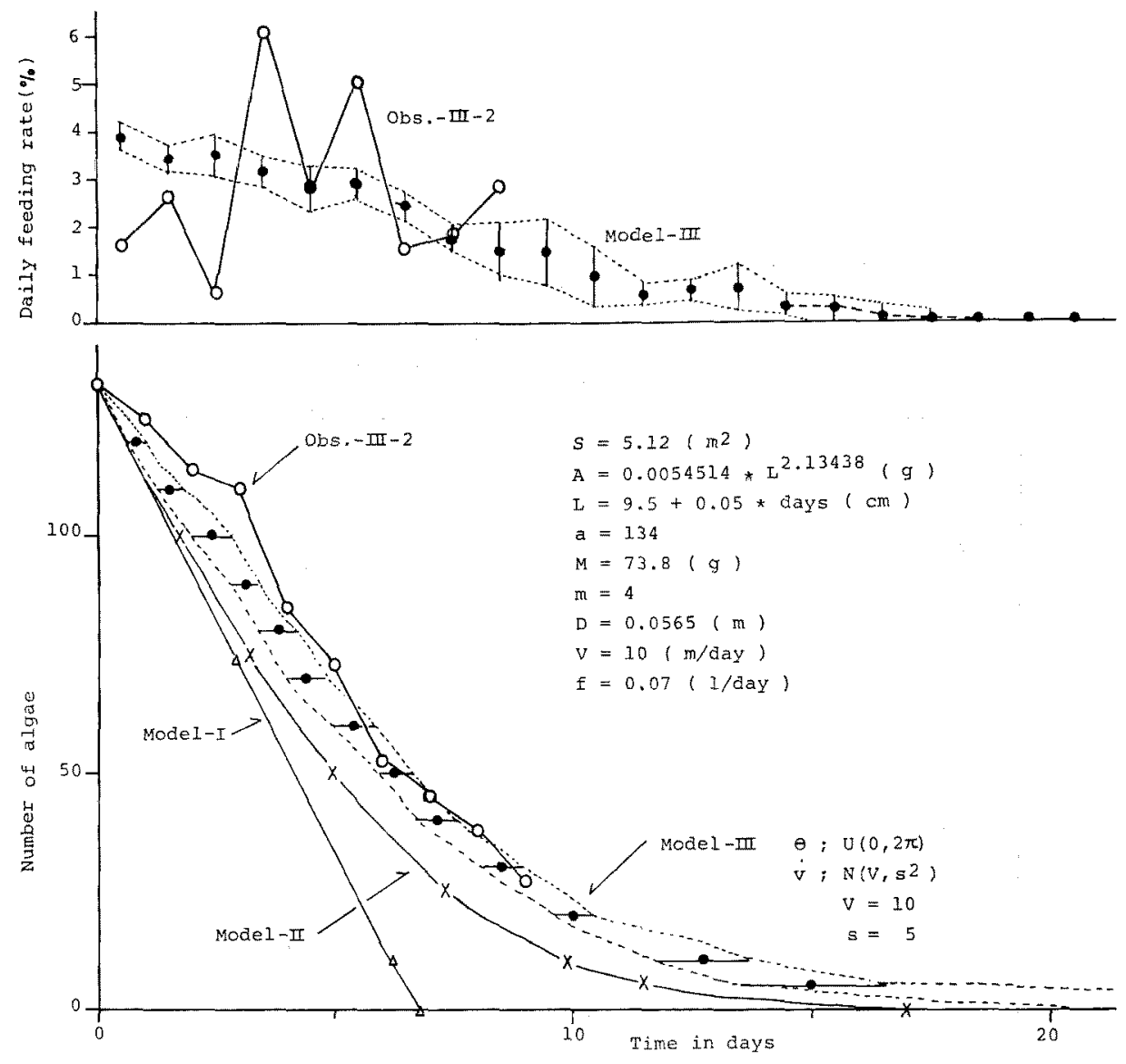

Fig. 6. Comparison of observation (Obs.-III-2; Jan., 1982) with model-I, model-II, and model-III.

\section{Results}

Naturally, more effective feeding with a constant daily feeding rate was shown in model-I. The feeding rate calculated in model-II decreased gradually as the algal density became low, even if a constant value of $f$ was given. Model-III provided the least effective feeding owing to the stochastic processes. The results of Obs.-III-1 and Obs.-III-2 were compared with those of the models in Fig. 5 and Fig. 6, respectively. On simulating, the chosen parameters such as $S, a$, $M, m$, and $D$ were the same as in the observations. The length of the algae was assumed to increase linearly during the experiments, and the wet weight of an alga was given by an empirical regression curve. ${ }^{4)}$ As for daily feeding rate, Fuji ${ }^{\text {) }}$ pointed out, in his observations related to the daily food amount of Laminaria japonica eaten by sea urchin Strongylocentritus intermedius, that the feeding rate $(5$ to $14 \%$ ) decreased as the sea urchin grew larger and fluctuated seasonally with high value in winter and early spring, and low in summer. In this simulation, however it was assumed to be a constant value of $7 \%$ a day for simplification. Mean velocity of moving sea urchins was assumed to be $10 \mathrm{~m} / \mathrm{day}$, by taking observation-II into consideration. Normal distribution adopted in model-III was characterized by a mean velocity of $10 \mathrm{~m} /$ day and a standard deviation of $5 \mathrm{~m} /$ day. Calculation in model-III was repeated five times.

As shown in Fig. 5 and Fig. 6, model-II simulated the grazing behavior to some extent and model-III gave a considerable fitness. However, abrupt changes in the grazing behavior could not be simulated. Thus, a detailed comparison between the results of daily feeding rate indicated the limited coincidence of the results.

\section{Discussions}

The better the model fits, the more time it takes for calculation. From this point of view, model- 

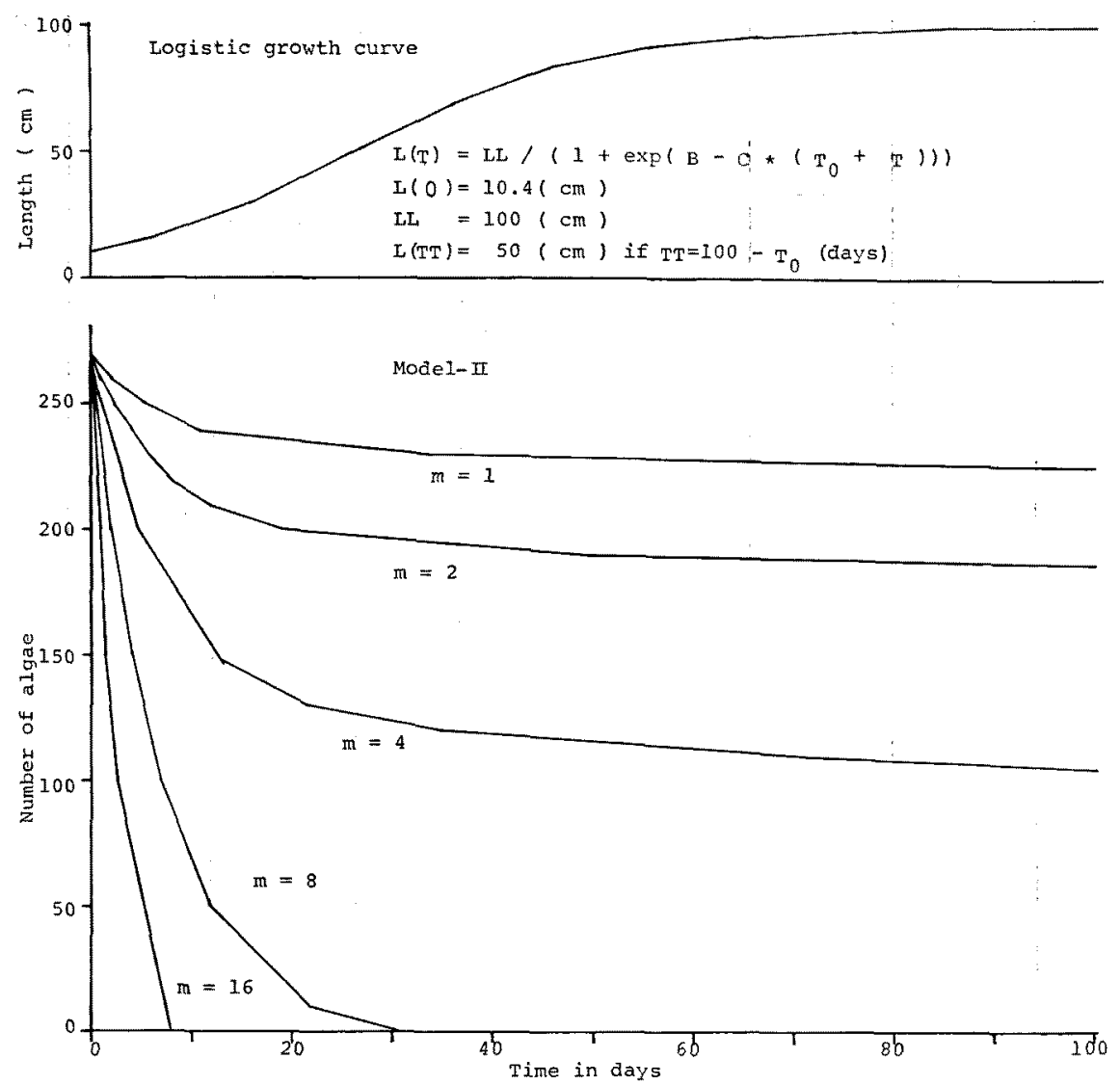

Fig. 7. Grazing effect on algal population with reference to the density of sea urchins.

TT is time for algal length to be half of its maximum. The growth curve is modeled after the natural growth of Undaria pinnatifida. The start of grazing corresponds to mid-winter when sea urchins feed vigorously and algae have not yet grown up. Thus, in this case, eight sea urchins are enough to eat up all the algae.

II is adopted under the following assumptions to make a rough estimate of density effects upon the extent of grazing.

1) Sea urchins graze the algae as described earlier in model-II, and values of the parameters are the same as those shown in Fig. 5 except for algal length increase, algae number, and sea urchins number.

2) The algal length increase is subject to logistic curve, which is given by

$$
L(T)=L L /\left(1+\exp \left(B+C \cdot\left(T_{0}+T\right)\right)\right.
$$

wherein $L L$ is the maximum length, $B$ and $C$ are constants, and $T_{0}$ is time for growing up to $L(0)$ that is the initial length of algae at the beginning of grazing.

3) Survival rate of the algae is to be evaluated after 100 days of grazing.

The logistic curve is determined by $L(0)$ once $L L$ and time for growing to half of $L L$ are known.
The maximum length is estimated at $100 \mathrm{~cm}$, and time to reach half of the maximum length at 100 days, by reference to the actual data..$^{4,6}$ Then, various cases are considered, that is, when the initial length of algae at the beginning of grazing, the density of algae, and the density of sea urchins are changing.

First, sea urchins density is variable while the initial length and algal density are constant. An example is given in Fig. 7, where the initial length is $10.4 \mathrm{~cm}$, the algal density is 52.5 individuals per $\mathrm{m}^{2}$, and the density of sea urchins ranges between 0.2 and 3.1 individuals per $\mathrm{m}^{2}$. In this case, the algae survival rate is evaluated to be zero when sea urchins density exceeds 1.6 individuals per $\mathrm{m}^{2}$.

Second, both densities of algae and sea urchins are changing, while the initial length of algae is constant. In this case, survival rate isopleths are 


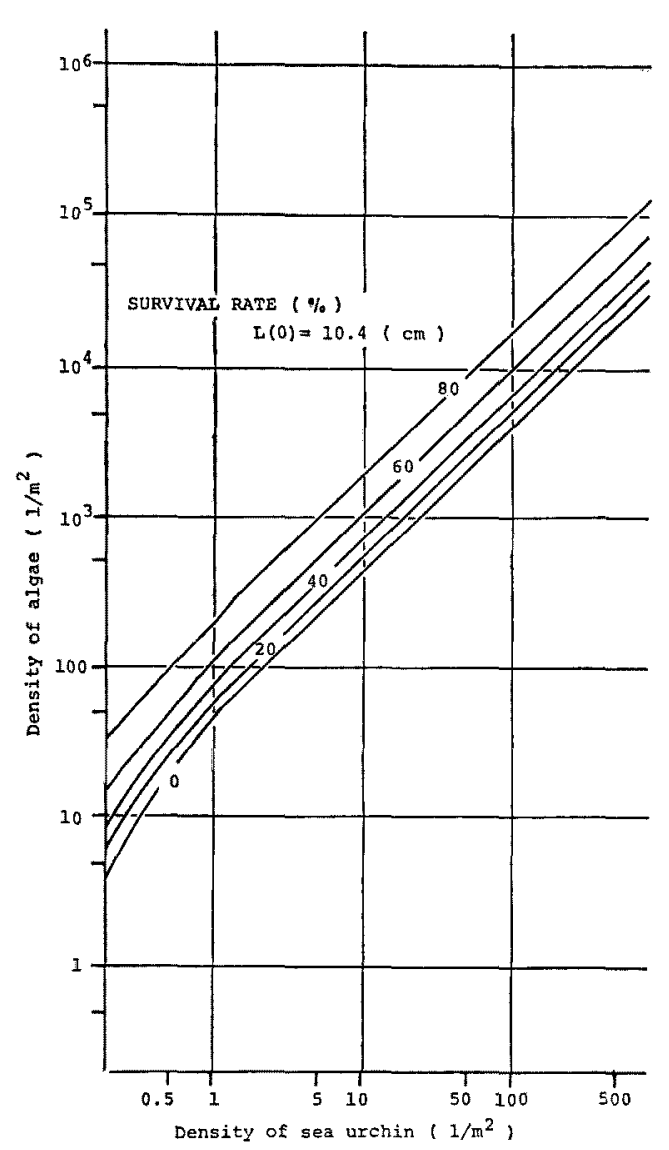

Fig. 8. Estimation of algal survival rate based on model-II. Survival rate depends on both densities of algae and sea urchins. Obviously, the zero isopleth is a critical boundary.

drawn, an example of which is in Fig. 8.

Finally, density-dependent relationships between sea urchins and algae through grazing are exemplified in Fig. 9. When sea urchins start on grazing the larger algae, the algal population can survive with the lower density and vice versa. Kawamura ${ }^{\text {?) }}$ reported that adult sea urchins density under natural condition in Hokkaido, was no more than 10 individuals per $\mathrm{m}^{2}$. Then, the algal density is expected to be more than 100 per $\mathrm{m}^{2}$ if sea urchins coexist with young algae of of $20 \mathrm{~cm}$ long.

\section{Acknowledgements}

The authors express sincere thanks to Dr. Y. Hénocque, Maison Franco-Japonaise, for his close inspection of manuscripts, Dr. H. Ogawa, Faculty of Agriculture, Tohoku University, for his encouraging advices, Mr. K. Yamada and Mr.

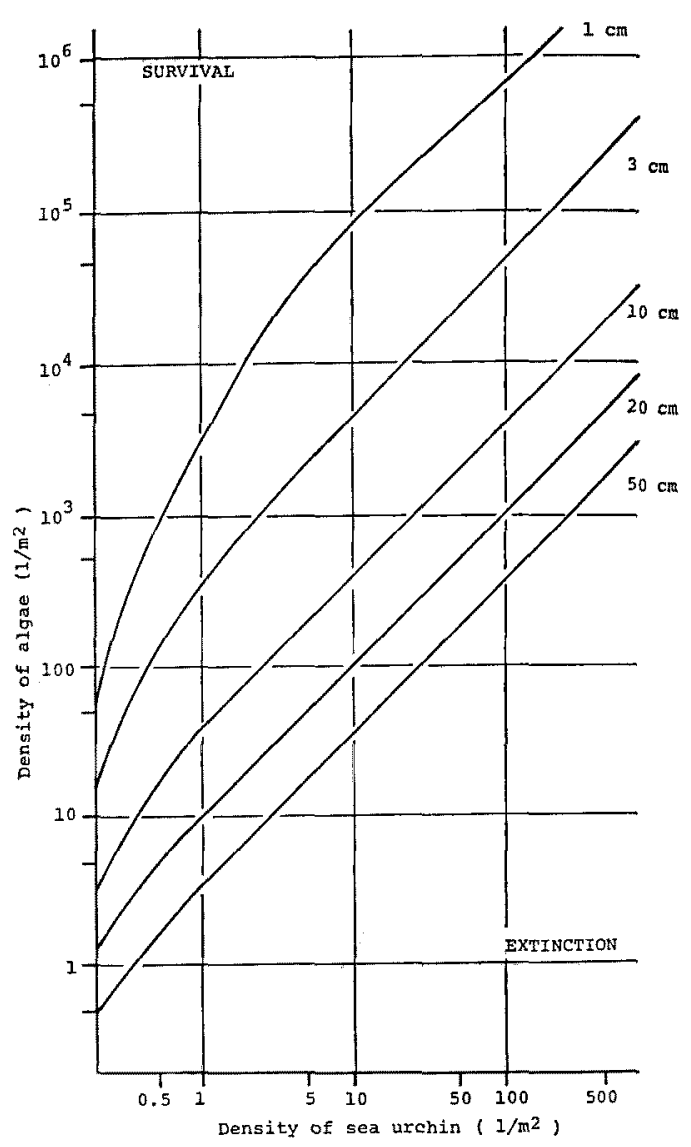

Fig. 9. Critical boundaries between survival and extinction of algal population.

Density-dependent relationship between sea urchins and algae through grazing is shown. These estimations are based on model-II.

K. Nishimura, former students of School of Fisheries Sciences, Kitasato University, for helping us with observations. This study was partly supported by the grant from the Ministry of Education, Science and Culture.

\section{References}

1) J. M. LoWrence: A. Rev. Oceanogr. Mar. Biol., 13, 213-286 (1975).

2) J. S. Pearse and A. H. Hines: Marine Biology, 51, 83-91 (1979).

3) J. Kittaka and Imamura: Marine Fouling, 3, 53-59 (1981).

4) T. Nonaka and Y. Iwahashi: The Aquiculture, 9, 299-236 (1962).

5) A. FUJI: Jap. J. Ecol., 12, 181-186 (1962).

6) H. NishIKaWA: The Aquiculture, 11, 175-186 (1963).

7) K. Kawamura: Sci. Rep. Hokkaido Fish. Exp. Stat., 5, 7-30 (1966). 